Table des matières du tome LXXV, fascicule 1

K. Nakano, Uniformity and classes of real valued function system Payes H. L. Bentley, Some Wallman compactifications of locally compact spaces 13-24 W. C. Chewning, Connectivity retracts of unicoherent Peano continua in $R^{n} \quad 25-27$ H. Potoczny, On a problem of Tamano . . . . . . . . . . . . . . 29-31

B. Fitzpatrick, Jr., A note on countable dense homogeneity . . . . . . 33-34

R. C. Freiwald, Images of Borel sets and $k$-analytic sets . . . . . . . . 35-46

D. Phillips, Real functions having graphs connected and dense in the plane $47-49$

G. R. Gordh, Jr., On decompositions of smooth continua . . . . . . . 51-60

M. J. Powers, Fixed point theorems for non-compact approximative ANR's 61-68

L. D. Nel and R. G. Wilson, Epireflections in the category of $T_{0}$-spaces $69-74$

R. Pol, On the position of the set of monotone mappings in function spaces $\quad 75-84$

R. H. Fox, Errata to the paper "On shape", Fundamenta Mathematicae

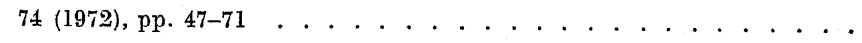

Les FUNDAMENTA MATHEMATICAE publierit, en langues des congrès internationaux, des tràvaux consacrés à la Théorie des Ensembles, Topologie, Fondements de Mathématiques, Fonctions Réelles, Algèbre Abstraite.

Chaque volume paraît en 3 fascicules

Adresse de la Rédaction et de l'Échange:

FUNDAMENTA MATHEMATICAE, Śniadeckich 8, Warszawa 1 (Pologne)

Le prix de ce fascicules est $4.00 \$$

Tous les volumes sont à obtenir par l'intermédiaire de

ARS POLONA - RUCH, Krakowskie Przedmieście 7, Warszawa 1 (Pologne)

DRUKARNIA UNIWERSYTETU JAGIELLONSKIEGO W KRAKOWIE

\section{Uniformity and classes of real valued function systems}

by

\author{
Kazumi Nakano (Brockport, N. Y.)
}

A set $\Phi$ of real valued functions on a space $S$ is called uniformly equi-continuous relative to a uniformity $\mathfrak{U}$ if for any $\varepsilon>0$, there is $U \in \mathfrak{U}$ such that $|\varphi(x)-\varphi(y)|<\varepsilon$ for all $\varphi \in \Phi$ whenever $(x, y) \in U$. Let $U(\Phi, \varepsilon)$ denote a, set of $(x, y) \in S \times S$ such that $|\varphi(x)-\varphi(y)|<\varepsilon$ for all $\varphi \in \Phi$. $\Phi$ is uniformly equi-continuous relative to a uniformity $\mathfrak{U}$ iff $U(\Phi, \varepsilon) \in \mathfrak{U}$ for all $\varepsilon>0$. $\mathfrak{U}$ is indeed the weakest uniformity which includes all of $U(\Phi, \varepsilon)$ such that $\varepsilon>0$ and $\Phi$ is uniformly equi-continuous relative to $\mathfrak{U}$ (see reference [11]). Conversely, there is the weakest uniformity for which every system in a given class of function systems, is uniformly equicontinuous. It is called a multiple equi-uniformity by a double system of functions in reference [9]. (In the rest of this paper, a real valued function and a system of real valued functions will be called a function and a function system respectively.) A function uniformity in reference [5], corresponds to a uniformity derived in this manner, from a class of function systems with some restrictions. However a uniformity can be derived from any class of function systems. Therefore theory of uniform structures can be developed by classes of function systems. Obviously, a same uniformity may be derived from different classes of function systems. This paper introduces an explicit definition of an equivalence relation between two such classes. Thus any property of uniform spaces can be discussed as a property preserved by the equivalence relation.

A purpose of this paper is to establish basic properties and apply them to classical important theorems. Some of them appear to be simpler with trivial proofs through this approach. Among them are Metrization theorem and $a$ characterization for a totally bounded uniformity by Cauchy nets (a uniformity is totally bounded iff every net has a Cauchy subnet). A typical proof of the latter involves a completion however the theorem can be proved directly without a completion. It is also easier to define a uniform structure and to recognize uniformly continuous functions and uniformly equi-continuous functions. Uniform continuity of a mapping between two uniform structures may be defined as follows. Fundåmenta Mathematicae, T. LXXV 
Suppose $M$ is a mapping form a space $S$ with a class $\mathfrak{U}$ of function systems to a space with a class $\mathfrak{B}$ of function systems. $M$ is uniformly continuous if for every $\Phi \in \mathfrak{B}$, a function system $\{\varphi \circ M ; \varphi \in \Phi\}$ is uniformly equicontinuous relative to a uniform structure given by $\mathfrak{A}$. A completion presented in this paper is explicit because a space is extended by additional elements rather than embedding it into a complete space. A class $\mathfrak{A}$ of function systems is called a unity if each $\Phi \in \mathfrak{A}$ contains bounded functions only and a set $\{\varphi(x) ; \varphi \in \Phi\}$ is bounded for every $x \in S$. In fact, for every class of function systems, there is a unity which is equivalent to it. A unity is called simple if each function system of it has only one function. If a class $\mathfrak{U}$ is equivalent to a simple unity then each $\Phi \in \mathfrak{A}$ has the property that, as a class which has only one function system, $\Phi$ is equivalent to a simple unity $\{\{\varphi\} ; \varphi \in \Phi\}$. This result can be applied to various classical theorems including Ascoli's theorem.

§ 1. Equivalence relation. Let $F$ be a set of all functions on a space $S$ and $\mathfrak{U}$ be a collection of subsets of $F$. For $\Phi \subseteq F$, one writes $\Phi>\mathfrak{U}$ if for any $\varepsilon>0$ there exist $\delta>0$ and a finite number of systems of functions $\Psi_{i}$, $i=1,2, \ldots, n$ in $\mathfrak{A}$ such that $|\psi(x)-\psi(y)|<\delta$ for all $\psi \in \bigcup_{i=1}^{n} \Psi_{i}$ implies $|\varphi(x)-\varphi(y)|<\varepsilon$ for all $\varphi \in \Phi$. Let $\mathfrak{B}$ be also a collection of subsets of $F$. One writes $\mathfrak{B} \succ \mathfrak{U}$ if $\Phi<\mathfrak{U}$ for all $\Phi \in \mathfrak{B}$. Define $\mathfrak{A} \sim \mathfrak{B}$ if $\mathfrak{U}\langle\mathfrak{B}$ and $\mathfrak{B} \succ \mathfrak{X}$ hold simultaneously. If $\mathfrak{A}$ or $\mathfrak{B}$ contains only $\Phi$ or $\Psi$ respectively, then notations $\Phi<\Psi, \Psi<\Phi$ and $\Phi \sim \Psi$ are used.

This relation is obviously transitive:

(1.1) $\mathfrak{A} く \mathfrak{B}$ and $\mathfrak{B} \zeta \mathfrak{C}$ implies $\mathfrak{A} \succ \mathfrak{C}$.

Also the inclusion order is preserved:

(1.2) $\mathfrak{A} \subset \mathfrak{B}$ implies $\mathfrak{A} \longrightarrow \mathfrak{B}$.

Hence $\mathfrak{A} \sim \mathfrak{B}$ is an equivalence relation.

Let $\overline{\mathfrak{U}}$ be the collection of all the subsets of $F$ such that $\Phi<\mathfrak{A}$. If $\Phi$ belongs to $\mathfrak{A}$ then $\Phi>\mathfrak{A}$, therefore

(i.3) $\mathfrak{U} \subset \overline{\mathfrak{X}}$.

This follows $\mathfrak{U} \succ \overrightarrow{\mathfrak{A}}$. By the definition of $\overline{\mathfrak{A}}, \overline{\mathfrak{A}} \longrightarrow \mathfrak{U}$ is obvious.

(1.4) $\mathfrak{U} \sim \overline{\mathfrak{X}}$.

The following properties are also obvious from the definition of $\overline{\mathfrak{X}}$.

(1.5) $\mathfrak{X} \sim \mathfrak{B}$ iff $\overline{\mathfrak{U}}=\overline{\mathfrak{B}}$.

(1.6) $\overline{\mathfrak{A}}=\overline{\overline{\mathfrak{A}}}$.

(1.7) $\Phi \in \mathfrak{U}$ then $\Psi \in \overline{\mathfrak{I}}$ for all $\Psi \subset \Phi$.

$\Phi, \Psi \in \overline{\mathfrak{I}}$ implies $\Phi \cup \Psi \in \overline{\mathfrak{A}}$.
If $\mathfrak{A}$ contains at most countable systems of functions, $\Phi_{n}$, $n=1,2,3, \ldots$, then define $\mathfrak{B}=\left\{\Psi_{n} ; \Psi_{n}=\bigcup_{i=1}^{n} \Phi_{i}, n=1,2,3, \ldots\right\} . \mathfrak{A}$ is equivalent to $\mathfrak{B}$, therefore one may assume the members of $\mathfrak{U}$ are chained if it is necessary.

(1.8) If $\mathfrak{A}$ is a countable collection of function systems then $\mathfrak{A}$ is equivalent to a countable chain.

The following property is obvious however it is useful.

(1.9) Let $\mathfrak{B}$ be a subcollection of $\mathfrak{A}$. Then $\mathfrak{A} \sim\{\Phi \cup \Psi ;(\Phi, \Psi) \in \mathfrak{X} \times \mathfrak{B}\}$.

$\S 2$. Unity. A system $\Phi$ of functions is called a unit if each function $\varphi \in \Phi$ is bounded and a set $\Phi(x)=\{\varphi(x) ; \varphi \in \Phi\}$ is bounded for every $x \in S$. A collection of units is called a unity. Any collection of systems of functions is equivalent to a unity. A following lemma precedes a proof of this theorem.

(2.1) Lemma. Let $\Phi$ be a set of functions. For $a \in S$, define $(f, a)(x)$ $=\operatorname{Min}\{1, \sup |\varphi(a)-\varphi(x)|\}$. Then $\{(f, a) ; a \in S\}$ is a unit and it is equivalent to $\Phi$.

Proof. Since each $(f, a)$ is bounded by 0 and 1 , a set $\{(f, a) ; a \in S\}$ is obviously a unit. For any $0<\varepsilon<1,|\varphi(x)-\varphi(y)|<\varepsilon$ for all $\varphi \in \Phi$ implies that $|(f, a)(x)-(f, a)(y)|<\varepsilon$ for all $a \in S$ because

$$
\begin{aligned}
|(f, a)(x)-(f, a)(y)| & =\left|\operatorname{Min}\left\{1, \sup _{\varphi \in \Phi}|\varphi(a)-\varphi(x)|\right\}-\operatorname{Min}\left\{1, \sup _{\varphi \in \Phi}|\varphi(a)-\varphi(y)|\right\}\right| \\
& \leqslant \operatorname{Min}\left\{1,\left|\sup _{\varphi \in \Phi}\right| \varphi(x)-\varphi(a)\left|-\sup _{\varphi \in \Phi}\right| \varphi(a)-\varphi(y) \mid\right\} \\
& \leqslant \operatorname{Min}\left\{1, \sup _{\varphi \in \Phi}|\varphi(x)-\varphi(y)|\right\}<\varepsilon .
\end{aligned}
$$

Conversely suppose $|(f, a)(x)-(f, a)(y)|<\varepsilon<1$ for all $a \in S$. Then

$$
|(f, x)(x)-(f, x)(y)|=|(f, x)(y)|=\operatorname{Min}\left\{1, \sup _{\varphi \in \Phi}|\varphi(x)-\varphi(y)|\right\}<\varepsilon .
$$

Therefore $|\varphi(x)-\varphi(y)|<\varepsilon$ for all $\varphi \in \Phi$. This implies that for any $0<\varepsilon<1$ $|(f, a)(x)-(f, a)(y)|<\varepsilon$ for all $a \in S$ iff $|\varphi(x)-\varphi(y)|<\varepsilon$ for all $\varphi \in \Phi$. Consequently $\Phi \sim\{(f, a) ; a \in S\}$.

(2.2) THEOREM. Any collection of systems of functions is equivalent to a unity such that every function of units is bounded by 0 and 1 .

Proof. For each $\Phi \in \mathfrak{U}$, there exists $\hat{\Phi}=\{(f, a) ; a \in S\}$ defined in (2.1). Let $\hat{\mathfrak{U}}$ be a collection of $\hat{\Phi}$ where $\Phi \in \mathfrak{U}$. Then $\mathfrak{U}$ is equivalent to $\hat{\mathfrak{A}}$.

§ 3. Simple unity. A unity is called simple if each unit is a single function. A simple unity of all the functions of units of $\mathfrak{U}$ is called simplicity of $\mathfrak{U}$ and denoted by $\mathfrak{A}^{*}$, namely $\mathfrak{A}^{*}=\{\{\varphi\} ; \varphi \in \Phi$ for some $\Phi \in \mathfrak{U}\}$. There 
is a characterization theorem of simple unity, which is proved after a sequence of the following lemmas.

(3.1) Lemia. Any net $\Delta$ has a subnet $\{\delta(\gamma) ; \gamma \in \Gamma\}$ such that $\varphi(\delta(\gamma))$; $\gamma \in \Gamma$ converges for any bounded real valued function $\varphi$ on $\Delta$.

Proof. For each $\delta \in \Delta, \Delta(\delta)$ denotes a subset of $\Delta$ such that $\Delta(\delta)$ $=\left\{\delta^{\prime} ; \delta^{\prime} \geqslant \delta\right\}$. Since $\Delta\left(\delta^{\prime}\right) \subseteq \Delta(\delta)$ iff $\delta \leqslant \delta^{\prime}$, there exists a maximal filter $\Gamma$ of subsets of $\Delta$, ordered by the inverse inclusion, such that $\Gamma$ contains all $\Delta(\delta)$ for $\delta \in \Delta$. By the axiom of choice, choose $\delta(\gamma)$ from each subset $\gamma$ of $\Gamma$. A net $\{\delta(\gamma) ; \gamma \in \Gamma\}$ is a subnet of $\Delta$ because for any $\delta \in \Delta$, there exists a member of $\Gamma$, namely $\Delta(\delta)$ such that $\gamma>\Delta(\delta)$ implies $\delta(\gamma) \geqslant \delta$. Let $\varphi$ be a bounded function. Suppose $\lim \sup \varphi(\delta(\gamma))$ and $\liminf \varphi(\delta(\gamma))$ are not equal and there exists a real number $\alpha$ between them. Let $\Delta_{1}$ and $\Delta_{2}$ be sets $\{\delta \in \Delta ; \varphi(\delta)>\alpha\}$ and $\{\delta \in \Delta ; \varphi(\delta)<\alpha\}$ respectively. $\lim \sup \varphi(\delta(\gamma))>\alpha$ implies that for any $\gamma \in \Gamma$, there exists $\gamma^{\prime} \in \Gamma$ such that $\varphi\left(\delta\left(\gamma^{\prime}\right)\right)>\alpha$ and $\gamma^{\prime}>\gamma$. Therefore $\gamma \cap \Delta_{1} \neq \emptyset$ for all $\gamma \in \Gamma$. This implies that $\Delta_{1}$ is a member of $\Gamma$. Similarly $\liminf \varphi(\delta(\gamma))<\alpha$ implies $\Delta_{2}$ is also a member of $\Gamma$. Since $\Gamma$ is a filter, $\Gamma$ also contains $\Delta_{1} \cap \Delta_{2}$. However $\Delta_{1}$ and $\Delta_{2}$ are disjoint, hence it contradicts the filter condition. Therefore $\lim \sup \varphi(\delta(\gamma))=\liminf \varphi(\delta(\gamma))$.

(3.2) LEMMA. Let $\mathfrak{B}$ be a simple unity. $\Phi>\mathfrak{B}$ implies that for any $\varepsilon>0$, there exists a finite subset $\Phi(\varepsilon) \subset \Phi$ and for any $\varphi \in \Phi$ there exists $\varphi^{\prime} \epsilon \Phi(\varepsilon)$ such that $\left|\varphi(x)-\varphi^{\prime}(x)\right|<\varepsilon$ for all $x \in S$.

Proof. Let $A(\varepsilon)$ be a maximal subset of $S$ with respect to a property: (1) $x, y \in A(\varepsilon), x \neq y$ implies $|\varphi(x)-\varphi(y)| \geqslant \varepsilon$ for some $\varphi \in \Phi$. Since $A(\varepsilon)$ is maximal, it follows that (2) for any $x \in S$, there exists $y \in A(\varepsilon)$ such that $|\varphi(x)-\varphi(y)|<\varepsilon$ for all $\varphi \in \Phi$. Suppose $A(\varepsilon)$ contains infinitely many elements, then it contains a sequence of distinct points. By (3.1), there exists a subnet of this sequence such that the image by any bounded function is a convergent net. Suppose $\left\{x_{\delta}\right\} \quad(\delta \in \Delta)$ is such a net. Since $\Phi<\mathfrak{B}$, there exist $\varepsilon^{\prime}>0$ and a finite number of functions $\psi_{1}, \psi_{2}, \ldots$ $\ldots, \Psi_{n} \in \mathfrak{B}$ such that $\left|\psi_{i}(x)-\psi_{i}(y)\right|<\varepsilon^{\prime}, i=1,2,3, \ldots, n$ implies $\mid \varphi(x)$ $-\varphi(y) \mid<\varepsilon$ for all $\varphi \in \Phi$. For $\varepsilon^{\prime}>0$, there exists $\delta \in \Delta$ such that $\mid \psi_{i}\left(x_{\delta}\right)-$ $-\psi_{i}\left(x_{\delta^{\prime}}\right) \mid<\varepsilon^{\prime}$ for all $i=1,2,3, \ldots, n, \delta^{\prime}>\delta$ thus $\left|\varphi\left(x_{\delta}\right)-\varphi\left(x_{\delta^{\prime}}\right)\right|<\varepsilon$ for all $\varphi \in \Phi, \delta^{\prime}>\delta$. However it contradicts the condition (1). Hence $A(\varepsilon)$ is a finite set. Since $\Phi$ is a unit, namely $\{\varphi(x) ; \varphi \in \Phi\}$ is bounded at each $x \in S$, there exists an interval which contains a set $\Phi(A(\varepsilon))=\{\varphi(y)$; for $\varphi \in \Phi, y \in A(\varepsilon)\}$. Divide this interval into a set of disjoint (except end points) intervals $\mathfrak{I}=\left\{I_{j}\right\}$ such that each $I_{j}$ has the length less than $\varepsilon$. Let $\mathfrak{I}(\varphi)$ be a subset of $\mathfrak{J}$ such that $I_{j} \in \mathfrak{I}(\varphi)$ iff $\varphi(y) \in I_{j}$ for some $y \in A(\varepsilon)$.

Define $\varphi E \varphi^{\prime}$ if $\mathfrak{I}(\varphi)=\Im\left(\varphi^{\prime}\right)$ and for each $y \in A(\varepsilon), \varphi(y)$ and $\varphi^{\prime}(y)$ belong to the same $I_{j}$ of $\Im(\varphi)$. Then $\varphi E \varphi^{\prime}$ is an equivalence relation. Let $\Phi(\varepsilon)$ be a set of representatives of equivalence classes. For any $\varphi \in \Phi$ there exists $\varphi^{\prime} \in \Phi(\varepsilon)$ such that $(3)\left|\varphi(y)-\varphi^{\prime}(y)\right|<\varepsilon$ for all $y \in A(\varepsilon)$. Since $\mathfrak{I}$ contains a finite number of intervals, the number of equivalence classes is finite, namely $\Phi(\varepsilon)$ is a finite set. For any $\varphi \in \Phi$, there exists $\varphi^{\prime} \in \Phi(\varepsilon)$ such that

$$
\left|\varphi(x)-\varphi^{\prime}(x)\right| \leqslant|\varphi(x)-\varphi(y)|+\left|\varphi(y)-\varphi^{\prime}(y)\right|+\left|\varphi^{\prime}(y)-\varphi^{\prime}(x)\right|<3 \varepsilon
$$

because (2) implies that there exists $y \in A(\varepsilon)$ such that $|\varphi(x)-\varphi(y)|<\varepsilon$ for all $\varphi \in \Phi$, and (3) implies $\left|\varphi(y)-\varphi^{\prime}(y)\right|<\varepsilon$ for all $y \in A(\varepsilon)$. Therefore $\left|\varphi(x)-\varphi^{\prime}(x)\right|<3 \varepsilon$ for all $x \in S$.

(3.3) LEMma. $\Phi \prec \mathfrak{B}$ implies $\Phi \sim \Phi^{*}$, where $\mathfrak{B}$ is a simple unity.

Proof. For $\varphi \in \Phi$, there exists $\varphi^{\prime} \in \Phi(\varepsilon)$ such that Lemma (3.2) holds. If $\left|\varphi^{\prime}(x)-\varphi^{\prime}(y)\right|<\varepsilon$ for all $\varphi^{\prime} \in \Phi(\varepsilon)$, then

$$
|\varphi(x)-\varphi(y)| \leqslant\left|\varphi(x)-\varphi^{\prime}(x)\right|+\left|\varphi^{\prime}(x)-\varphi^{\prime}(y)\right|+\left|\varphi^{\prime}(y)-\varphi(y)\right|<3 \varepsilon .
$$

Since $\Phi(\varepsilon)$ is a finite set, this implies $\Phi>\Phi^{*}$. The other direction of order is always true, therefore $\Phi \sim \Phi^{*}$.

(3.4) THEOREM. If $\mathfrak{A} \sim \mathfrak{B}$ for some simple unity $\mathfrak{B}$, then $\mathfrak{A} \sim \mathfrak{Y}^{*}$.

Proof. $\mathfrak{A} \sim \mathfrak{B}$ implies that $\Phi \ \mathfrak{B}$ for all $\Phi \in \mathfrak{A}$. By the previous lemma, this implies $\Phi \sim \Phi^{*}$ for all $\Phi \in \mathfrak{U}$. Therefore $\mathfrak{A} \sim \mathfrak{U}^{*}$.

§ 4. Single unity. A unity is called single if it contains only one unit.

(4.1) THEOREM. A unity of countable units is equivalent to a single unity.

Proof. Let $\mathfrak{U}$ be $\{\Phi(n) ; n=1,2,3, \ldots\}$. One may assume each unit is a set of functions bounded by 0 and 1 . For each triple $(n, \varphi, a)$ $\epsilon\{n\} \times \Phi(n) \times S$, define a function: $\varphi(n, a)(x)=1 / n|\varphi(x)-\varphi(a)|$. Let $\Phi$ bo a unit of all such functions, namely $\Phi=\{\varphi(n, a)$; for $(n, \varphi, a) \in\{n\} \times$ $\times \Phi(n) \times S, \quad n=1,2,3, \ldots\} . \quad|\varphi(m, a)(x)-\varphi(m, a)(y)|<1 / n \cdot \varepsilon$ for all $\varphi(m, a) \in \Phi$ implies that

$$
|\varphi(x)-\varphi(y)|=n \varphi(n, y)(x)=n|\varphi(n, y)(x)-\varphi(n, y)(y)|<\varepsilon
$$

for all $\varphi \in \Phi(n)$. Therefore $\Phi(n)>\Phi$. Since $n$ is arbitrary, $\mathfrak{A} \ \Phi$.

Conversely for $\varepsilon>0$, choose $n_{0}$ such that $1 / n_{0}<\varepsilon$, then

$|\varphi(n, a)(x)-\varphi(n, a)(y)| \leqslant 1 / n|\varphi(x)-\varphi(y)|<1 / n<1 / n_{0}<\varepsilon$ for all $n>n_{0}$.

Suppose $|\varphi(x)-\varphi(y)|<\varepsilon$ for all $\varphi \in \bigcup \Phi(n), n=1,2,3, \ldots, n_{0}$ then

$$
|\varphi(n, a)(x)-\varphi(n, a)(y)| \leqslant 1 / n|\varphi(x)-\varphi(y)|<\varepsilon \quad \text { for all }
$$

$$
(n, \varphi, a) \epsilon\{n\} \times \Phi(n) \times S, n=1,2,3, \ldots, n_{0} .
$$

Therefore $|\varphi(x)-\varphi(y)|<\varepsilon$ for all $\varphi \in \cup \Phi(n) n=1,2,3, \ldots, n_{0}$ implies

$$
|\varphi(n, a)(x)-\varphi(n, a)(y)|<\varepsilon \quad \text { for all } \varphi(n, a) \in \Phi,
$$

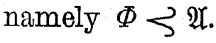


(4.2) THEOREM. A unity $\mathfrak{A}$ is equivalent to a single unity iff there exists a countable number of units $\Phi(n) \in \mathfrak{A}, n=1,2,3, \ldots$ such that $\mathfrak{A}$ $\sim\{\Phi(n) ; n=1,2,3, \ldots\}$.

Proof. Suppose that $\mathfrak{A}$ is equivalent to $\{\Phi(n) ; 1,2,3, . .$.$\} for some$ $\Phi(n) \in \mathfrak{U}, n=1,2,3, \ldots$ Then by (4.1) $\mathfrak{U}$ is equivalent to a single unity. If $\mathfrak{A}$ is equivalent to a single unity $\{\Phi\}$ then for each $n$, there exist a finite number of units $\Psi(m, n) \in \mathfrak{A}, m=1,2,3, \ldots, m_{0}$ and $\delta>0$ such that $|\psi(x)-\psi(y)|<\delta$ for all $\psi \in \bigcup \Psi(m, n) ; \quad m=1,2,3, \ldots, m_{0}$ implies $|\varphi(x)-\varphi(y)|<1 / n$. Let $\mathfrak{B}$ be a set of all such units: $\{\Psi(m, n) ; n, m\}$, $\mathfrak{B}$ is countable and equivalent to $\mathfrak{A}$.

$\S 5$. Convergence. A net $x(\delta) \in S$ with a directed set $\Delta$ is called semiconvergent with respect to $\mathfrak{A}$ if $\varphi(x(\delta)), \delta \in \Delta, \varphi \in \Phi$, converges uniformly on $\Phi$, for every $\Phi \in \mathfrak{A}$. A net $x(\delta), \delta \in \Delta$ is called convergent to $x$ if $\varphi(x(\delta))$ converges to $\varphi(x)$ uniformly on $\Phi$, for every $\Phi \in \mathfrak{A}$.

(5.1) Semi-convergence (convergence) is preserved by equivalence relation of unities.

The proof of (5.1) is a routine check of the definition of equivalence relation of unities.

(5.2) $\mathfrak{U} \sim \mathfrak{A}^{*}$ iff any net $x(\delta) \delta \in \Delta$ has a subnet $x(\delta(\gamma)), \gamma \in \Gamma$ which is semi-convergent with respect to $\mathfrak{A}$.

Proof. Lemma (3.1) shows that any net has a subnet which is semiconvergent with respect to $\mathfrak{A}^{*}$. It is also semi-convergent with respect to $\mathfrak{A}$ if $\mathfrak{A}$ is equivalent to $\mathfrak{A}^{*}$. To prove the converse direction, one may adopt the proof of (3.2) with a slight change. Let $A(\varepsilon)$ be a maximal set with a property: $x, y \in A(\varepsilon), x \neq y$ implies $|\varphi(x)-\varphi(y)| \geqslant \varepsilon$ for some $\varphi \in \Phi$. Suppose $A(\varepsilon)$ contains a sequence of distinct elements, then by the assumption, there exists a subnet $x(\delta), \delta \in \Delta$, which is semi-convergent with respect to $\mathfrak{A}$, namely, except finitely many distinct elements,

$$
\left|\varphi(x(\delta))-\varphi\left(x\left(\delta^{\prime}\right)\right)\right|<\varepsilon \quad \text { for all } \varphi \in \Phi .
$$

However this contradicts the assumption, hence $A(\varepsilon)$ contains finitely many elements. This is the only place where the assumption $(\Phi<\mathfrak{B}$ for some simple unity $\mathfrak{B}$ ) is used in the proof of Lemma (3.2) and the following Theorem (3.3). Therefore the assumption can be replaced by the assimption of this theorem to prove $\Phi \sim \Phi^{*}$ and $\mathfrak{A} \sim \mathfrak{A}^{*}$.

$\S$ 6. Application to uniformities. Define $(x, y) \in U(\Phi, \varepsilon)$ if $|\varphi(x)-\varphi(y)|<\varepsilon$ for all $\varphi \in \Phi$. Let $U(\Phi, \varepsilon)(x)$ be a set of all $y$ such that $(x, y) \in U(\Phi, \varepsilon)$. Let $\mathscr{B}$ be a set of all possible finite intersections of $U(\Phi, \varepsilon),(\Phi, \varepsilon) \epsilon \mathfrak{U} \times\{\varepsilon ; \varepsilon>0\}$ $\Re$ satisfies the base conditions, that is, (1) a finite intersection of members of $\mathcal{B}$ is again a member of $\beta$, and (2) $y \in \bigcap_{i=1}^{n} U\left(\Phi_{i}, 1 / 2 \varepsilon_{i}\right)(z)$ and $z \epsilon \bigcap_{i=1}^{n} U\left(\Phi_{i}, 1 / 2 \varepsilon_{i}\right)(x)$ implies $y \in \bigcap_{i=1}^{n} U\left(\Phi_{i}, \varepsilon_{i}\right)(x)$. Therefore there exists a uniformity $\mathfrak{U}(\mathfrak{U})$ with a base $\mathfrak{B}$.

(6.1) If $\mathfrak{A}$ contains any finite union of its units, then a base $\mathfrak{B}$ of $\mathfrak{U}(\mathfrak{U})$ is $\{U(\Phi, \varepsilon) ;(\Phi, \varepsilon) \in \mathfrak{A} \times\{\varepsilon ; \varepsilon>0\}\}$.

Proof. The following inclusions prove the proposition.

$$
U\left(\bigcup_{i=1}^{n} \Phi_{i}, \operatorname{Min} \varepsilon_{i}\right) \subseteq \bigcap_{i=1}^{n} U\left(\Phi_{i}, \varepsilon_{i}\right) \subseteq U\left(\bigcup_{i=1}^{n} \Phi_{i}, \operatorname{Max} \varepsilon_{i}\right) .
$$

(6.2) $\mathfrak{U} \ \mathfrak{B}$ iff $\mathfrak{U}(\mathfrak{U}) \subseteq \mathfrak{U}(\mathfrak{B})$, thus $\mathfrak{A} \sim \mathfrak{B}$ iff $\mathfrak{U}(\mathfrak{U})=\mathfrak{U}(\mathfrak{B})$.

The proof of this proposition is again a routine check of the definition of equivalence of unities.

(6.3) Let $\mathcal{B}$ be $\{U(\Phi, \varepsilon) ;(\Phi, \varepsilon) \in \overline{\mathfrak{A}} \times\{\varepsilon ; \varepsilon>0\}\}$. Then $\mathcal{B}$ is a base of $\mathfrak{U}(\mathfrak{U})(\overline{\mathfrak{A}}=\{\Phi ; \Phi \zeta \mathfrak{U}\})$.

Proof. By (6.1), $\mathfrak{B}$ is a base of $\mathfrak{U}(\overline{\mathfrak{U}})$. Since $\mathfrak{A} \sim \overline{\mathfrak{A}}, \mathfrak{B}$ is also a base of $\mathfrak{U}(\mathfrak{U})$.

(6.4) $\mathfrak{U}(\mathfrak{U})$ has a countable base iff $\mathfrak{A}$ is equivalent to a unity of countable units.

Proof. By (1.8), one may assume $\mathfrak{A}$ is a chain of countable units. Therefore $\mathfrak{B}=\{U(\Phi, 1 / n) ;(\Phi, 1 / n) \epsilon \mathfrak{A} \times\{1 / n ; n=1,2,3, \ldots\}\}$ is a base of $\mathfrak{U}(\mathfrak{U})$ and obviously $\mathcal{B}$ is countable. Conversely suppose $\{U(n)$; $n=1,2,3, \ldots\}$ is a countable base of $\mathfrak{U}(\mathfrak{U})$. For each $U(n)$, there exists $U(\Phi(n), \varepsilon(n)) ;(\Phi(n), \varepsilon(n)) \epsilon \overline{\mathfrak{Y}} \times\{\varepsilon ; \varepsilon>0\}$ such that $U(\Phi(n), \varepsilon(n)) \subset U(n)$ because $\{U(\Phi, \varepsilon) ;(\Phi, \varepsilon) \in \overline{\mathfrak{U}} \times\{\varepsilon ; \varepsilon>0\}\}$ is also a base of $\mathfrak{U}(\mathfrak{U})$. Let $\mathfrak{B}$ be a unity of all such $\Phi(n) ; n=1,2,3, \ldots$ Obviously $\mathfrak{B}$ is countable and $\mathfrak{B}$ is also equivalent to $\overline{\mathfrak{U}}$ because for any $\Phi \in \overline{\mathfrak{Y}}$ and $\varepsilon>0$, there exists $U(n)$ such that $U(\Phi(n), \varepsilon(n)) \subseteq U(n) \subseteq U(\Phi, \varepsilon)$. Hence $\mathfrak{B}$ is also equivalent to $\mathfrak{A}$.

A semi-convergent (convergent) net with respect to $\mathfrak{A}$ is a Cauchy net (convergent net) with respect to $\mathfrak{U}(\mathfrak{U})$ and vice versa. Since a totally bounded uniformity is characterized by the induced uniformity from a simple unity, Theorem (5.2) can be applied to the well known theorem: a uniformity is totally bounded iff any net has a Cauchy subnet.

$\$ 7$. Completion. A space $S$ is called complete with respect to a unity $\mathfrak{Z}$ if every semi-convergent net is convergent. Obviously this definition is compatible with the completeness of a uniform space $S$ with the induced uniformity $\mathfrak{H}(\mathfrak{A})$. If $S$ is not complete, then there exists a completion of $S$ in the following sense. 
Defrntrion. A space $\tilde{S}$ with a unity $\tilde{\mathfrak{U}}$ is a completion of $S$ (with a unity $\mathfrak{U}$ ), if the following conditions are satisfied:

(1) $\mathbb{S} \subseteq \tilde{S .}$

(2) $\widetilde{S}$ is complete with respect to $\tilde{\mathfrak{A}}$.

(3) $\tilde{\mathfrak{U}}$ agrees with $\mathfrak{A}$ on $S$, that is, there exists a one to one correspondence $\widetilde{T}$ from $\mathfrak{H}^{*}$ on to $\widetilde{\mathfrak{A}}^{*}$, such that (i) for every $\varphi \in \mathfrak{A}^{*}, \varphi(x)=\widetilde{T}(\varphi)(x)$ for all $x \in S$, (ii) $\widetilde{\Phi} \in \widetilde{\mathfrak{I}}$ iff $\widetilde{\Phi}=\{\widetilde{T}(\varphi) ; \varphi \in \Phi\}$ for $\Phi \in \mathfrak{A}$.

(4) For any $x \in \tilde{S}$, there exists a net in $S$ which converges to $x$ with respect to $\tilde{\mathfrak{A}}$.

(5) For any distinct $x \in \tilde{\mathbb{S}}-S$ and $y \in \tilde{\mathbb{S}}$, there exists $\tilde{\varphi} \in \tilde{\mathfrak{A}}^{*}$ such that $\tilde{\varphi}(x) \neq \tilde{\varphi}(y)$.

A completion is denoted by either appair $(\widetilde{\mathcal{S}}, \tilde{\mathfrak{U}})$ or a triple $(\widetilde{\mathcal{S}}, \tilde{\mathfrak{A}}, \widetilde{T})$.

A completion is usually constructed by imbedding a space into a complete space and taking the closure of the image. However a method used here is constructive. First, a function on $\mathfrak{A}^{*}$ is defined associated with a net in $S$, which is semi-convergent but not convergent. A union of all such functions and the original space $S$ is a completion. A unity on this space is defined in a natural way. The detail follows.

Let $x_{\delta} \in S(\delta \in \Delta)$ be a net which is semi-convergent but not convergent. Since $x_{\delta} \in S(\delta \in \Delta)$ is semi-convergent $\lim _{\delta \in \Delta} \varphi\left(x_{\delta}\right)$ always exists for every $\varphi \in \mathfrak{U}^{*}$. Define $f(\varphi)=\lim _{\delta \in \Delta} \varphi\left(x_{\delta}\right)$ for $\varphi \in \mathfrak{A}^{*}$. Let $\widetilde{\delta}$ be a union of $S$ and all such functions defined above. Each $\varphi$ of $\mathfrak{A}^{*}$ ean be extended to $\tilde{\varphi}$ a function on $\tilde{S}$ in a natural way: Define

$$
\begin{aligned}
& \tilde{\varphi}(x)=\varphi(x) \text { for all } \quad x \in S, \\
& \tilde{\varphi}(f)=f(\varphi) \text { for all } f \in \tilde{S}-S \text {. }
\end{aligned}
$$

Such an extension of functions of $\mathfrak{U}^{*}$ is denoted by a mapping $\widetilde{T}: \widetilde{T}(p)=\tilde{p}$. Also define a unit $\widetilde{\Phi}=\{\widetilde{T}(\varphi) ; \varphi \in \Phi\}$ for $\Phi \in \mathfrak{U}$ and a unity $\widetilde{\mathfrak{I}}=\{\widetilde{\Phi} ; \Phi \in \mathfrak{U}\}$.

(7.1) THeOReMr. A triple $\left(\widetilde{\mathbb{S}^{\prime}}, \tilde{\mathfrak{A}}, \widetilde{T}\right)$ is a completion of (S, $\left.\mathfrak{N}\right)$.

Proof. Obviously a triple is constructed to satisfy conditions (1) and (3). (4) By the definition of $f \in \tilde{S}-S, \widetilde{\varphi}(f)=f(\varphi)=\lim _{\delta \in \Delta} \varphi\left(x_{0}\right)$ for $\varphi \in \mathfrak{A}^{*}$, and a net $\varphi\left(x_{\delta}\right)$ converges to $\tilde{\varphi}(f)$ uniformly on every $\Phi \in \mathfrak{\Phi} \in \mathbb{\mathfrak { U }}$. Hence a net $x_{\delta} \in S(\delta \in \Delta)$ converges to $f$ with respect to $\tilde{\mathfrak{U}}$. (5) Since members of $\tilde{S}-S$ are functions on $\mathfrak{A}^{*}, f \neq g \in \widetilde{S}-S$ implies that $f(\varphi) \neq g(\varphi)$ for some $\varphi \in \mathfrak{A}^{*}$, thus $\tilde{\varphi}(f) \neq \tilde{\varphi}(g)$ for some $\tilde{\varphi} \in \widetilde{\mathfrak{A}}^{*}$. Suppose that for $f \in \widetilde{S}-S$, there exists $x \in \mathbb{S}$ such that $\tilde{\varphi}(f)=\tilde{\varphi}(x)$ for all $\tilde{\varphi} \in \tilde{\mathfrak{A}}^{*}$. Then every net of $S$ which converges to $f$, must converge to $x$. Howerer by the definition of $f$, there exists a net of $S$, which converges to $f$ but to none of elements of $S$.

(2) Let $y_{\delta} \in \widetilde{\mathcal{S}}(\delta \in \Delta)$ be semi-convergent with respect to $\tilde{\mathfrak{U}}$. Define a function $f$ on $\mathfrak{A}^{*}$ by $f(\varphi)=\lim _{\delta \in \Delta} \tilde{\varphi}\left(y_{\delta}\right)$ where $\tilde{\varphi}=\widetilde{T}(\varphi)$. Then for a pair $(\varepsilon, \Phi), \varepsilon>0, \Phi \in \mathfrak{U}$, there exists $y_{\delta}$ such that $\left|f(\varphi)-\tilde{\varphi}\left(y_{\delta}\right)\right|<\varepsilon / 2$ for all $\varphi \in \Phi$. By (4), there exists $x \in S$ such that $\left|\tilde{\varphi}\left(y_{\delta}\right)-\varphi(x)\right|<\varepsilon / 2$ for all $\varphi \in \Phi$, thus for a pair $(\varepsilon, \Phi)$, there exists $x \in S$ such that $|f(\varphi)-\varphi(x)|<\varepsilon$ for all $\varphi \in \Phi$. A set $\Gamma=\{(\varepsilon, \Phi) ; \varepsilon>0, \Phi \in \mathfrak{U}\}$ is a directed set provided with an order which is defined by $\left(\varepsilon^{\prime}, \Psi\right) \geqslant(\varepsilon, \Phi)$ if $\varepsilon \geqslant \varepsilon^{\prime}$ and $\Phi \subset \Psi$. A set of elements of $S$ selected above, associated with members of $\bar{T}$, is a net which is semi-convergent. Let it be denoted by $\left\{x_{\gamma}\right\}(\gamma \in T)$. Since $\lim \varphi\left(x_{\gamma}\right)$ $=f(\varphi)$ and convergence is uniform on each $\Phi \in \mathfrak{A}, f$ is a member of $\tilde{S}-S$ provided that a net $\left\{x_{\gamma}\right\}(\gamma \in \Gamma)$ does not converge to an element of $S$. Then obviously a net $\left\{y_{\delta}\right\}(\delta \in \Delta)$ also converges to $f$ with respect to $\overline{\mathfrak{A}}$. If a net $\left\{x_{\gamma}\right\}(\gamma \in \Gamma)$ converges to an element $x$ of $S$, then a net $\left\{y_{\delta}\right\}(\delta \in \Delta)$ should also converge to $x$ because $\lim _{\gamma \in \Gamma} \varphi\left(x_{\gamma}\right)=\varphi(x)=f(\varphi)$ and $\lim _{\delta \in \Gamma} \tilde{\varphi}\left(y_{\delta}\right)$ $=f(\varphi)$ uniformly on $\Phi$.

(7.2) Lemia. Let $(\widetilde{\mathcal{S}}, \widetilde{\mathfrak{N}})$ be a completion of $(\mathcal{S}, \mathfrak{H})$. Any net of $S$, which converges to an element of $\widetilde{S}-S$, converges to the unique element.

Proof. Suppose a net converges to $x \in \tilde{S}-S$ and $y \in \tilde{S}$ and $x \neq y$. Then $\tilde{\varphi}(x)=\tilde{\varphi}(y)$ for all $\tilde{\varphi} \in \tilde{\mathfrak{A}}^{*}$. However this contradicts the condition (5).

(7.3) THEOREM. Let $(\widetilde{\mathcal{S}}, \tilde{\mathfrak{A}}, \widetilde{T})$ and $\left(\mathcal{S}^{\prime}, \mathfrak{A}^{\prime}, T^{\prime}\right)$ be completions of $(\mathcal{S}, \mathfrak{A})$. Then there exists an one to one mapping $T$ from $\widetilde{S}$ on to $S^{\prime}$ such that $\widetilde{T}(\varphi)(\widetilde{x})$ $=T^{\prime}(\varphi)(T(\widetilde{x}))$ for all $\widetilde{x} \epsilon \widetilde{S}$ and $\varphi \in \mathfrak{A}^{*}$.

Proof. By the condition (4) of completion, for any $\tilde{x} \in \tilde{S}-S$, there exists a net $\left\{x_{\delta}\right\}(\delta \in \Delta)$ of $S$, which converges to $\tilde{x}$. By (7.2) this net does not converge to any elements of $S$. Since $S^{\prime}$ is also a completion of $S$, a net $\left\{x_{\delta}\right\}$ converges to $x^{\prime} \in S^{\prime}-S$. Again by (7.2), $x^{\prime}$ is determined uniquely. Any net $\left\{y_{\delta}\right\}(\delta \in \Delta)$ of $S$ which converges to $\widetilde{x}$ also converges to $x^{\prime}$ with respect to $\mathfrak{A}^{\prime}$ because $\Gamma$ a set of ordered pairs $\left\{\left(x_{\delta}, y_{\delta}\right),\left(y_{\delta}, x_{\delta}\right), \delta \in \Delta\right\}$ is a directed set provided with an order:

$$
\begin{aligned}
\left(x_{\delta}, y_{\delta}\right) \leqslant\left(x_{\delta^{\prime}}, y_{\delta^{\prime}}\right), \quad\left(x_{\delta}, y_{\delta}\right) \leqslant\left(y_{\delta^{\prime}}, x_{\delta^{\prime}}\right), \quad\left(y_{\delta}, x_{\delta}\right) \leqslant\left(x_{\delta^{\prime}}, y_{\delta^{\prime}}\right) & \\
& \text { and }\left(y_{\delta}, x_{\delta}\right) \leqslant\left(y_{\delta^{\prime}}, x_{\delta^{\prime}}\right)
\end{aligned}
$$

if $\delta \leqslant \delta^{\prime}$, and a net $\left\{x_{\delta}\right\}(\delta \in \Delta)$ and a net $\left\{y_{\delta}\right\}(\delta \in \Delta)$ are subnets of a net $\left\{z_{\gamma}\right\} \quad(\gamma \in T)$ which defined by $z_{\gamma}=$ the first coordinate of $\gamma$. Hence regardless of the choice of a net, $x^{\prime}$ is uniquely determined. Define $T(\widetilde{x})=x^{\prime}$ 
for $\tilde{x} \in \tilde{S}-S$ and $T(x)=x$ for $x \in S$. Since for any $\tilde{x} \in \tilde{S}-S$ and $\varepsilon>0$, there exists $x \in \mathbb{S}$ such that

$$
\begin{gathered}
|\tilde{T}(\varphi)(\tilde{x})-\tilde{T}(\varphi)(x)|<\varepsilon / 2 \text { and } \mid T^{\prime}(\varphi)\left(T(\tilde{x})\left|-T^{\prime}(\varphi)(x)\right|<\varepsilon / 2\right. \\
\text { for all } \varphi \in \mathfrak{A}^{*}, \\
\mid \widetilde{T}(\varphi)(\tilde{x})-T^{\prime}(\varphi)\left(T(\tilde{x}) \mid<\varepsilon \text { for all } \varphi \in \mathfrak{Y}^{*} .\right.
\end{gathered}
$$

A mapping $T$ is obviously one to one and onto.

A mapping $T$ defined in (7.3) is uniform isomorphism with respect to the induced uniformities. Therefore (7.3) proves the uniqueness of the completion up to the uniform isomorphism. A reason why the condition (5) is added is to garantee the uniqueness of the completion no matter the original space $S$ is a Hausdorff space or not, in other words, members of $\mathfrak{U}^{*}$ may not separate elements of $S$.

§ 8. Application to metric uniformities. Let $\Phi$ be a unit. Define a metric $m$ by $m(x, y)=\sup |\varphi(x)-\varphi(y)|$. Then a metric uniformity $\mathfrak{U}(m)$ is the

induced uniformity from a single unity $\{\Phi\}$. Conversely any metric uniformity is the induced uniformity from a single unity because a set of functions, $\{\varphi(y) ; \varphi(y)(x)=\operatorname{Min}\{n(x, y), 1\} y \in S\}$ is a unit which induces a metric uniformity $\mathfrak{I}(n)$.

By (4.1), any unity of countable units is equivalent to a single unity, therefore Theorem (6.4) implies a classical theorem: a uniformity is metrizable iff it has a countable base.

$\S$ 9. Uniform continuous mappings. Let $(S, \mathfrak{U})$ and $(R, \mathfrak{B})$ be spaces with unities $\mathfrak{A}$ and $\mathfrak{B}$ respectively and let $M$ be a mapping from $S$ to $R$. By composing $M$ to each function of a unit $\Phi$ of $\mathfrak{B}$, a unit on $S$ is obtained, which is denoted by $\Phi M$, that is, $\Phi M=\{\varphi M ; \varphi \in \Phi\}$. A unity which consists of all these units $\Phi M, \Phi \in \mathfrak{B}$ is denoted by $\mathfrak{B} M$.

Definition. A mapping $M$ from $(S, \mathfrak{A})$ to $(R, \mathfrak{B})$ is called uniformly continuous if $\mathfrak{B M}>\mathfrak{U}$.

Obviously this definition is compatible with a definition of a uniformly continuous mapping with respect to the induced uniformities. The following theorem is self-explanatory from the definition.

(9.1) Theorem. Let $M$ be a mapping from a space $S$ to a space $R$ with a unity $\mathfrak{B}$. Then $\mathfrak{B} M$ is the weakest unity on $S$ such that $M$ is uniformly continuous.

By (6.2), a weaker unity induces a weaker uniformity, thus (9.1) implies that the induced uniformity from $\mathfrak{B} M$ is the weakest uniformity on the domain space such that $M$ is uniformly continuous.
On the other hand, if a mapping $M$ is defined from a space $S$ equipped with a unity $\mathfrak{A}$, to a space $R$, then it is possible to define a strongest unit $\mathfrak{B}$ on $R$ such that $M$ is uniformly continuous. Obviously the induced uniformity from such a unity is the strongest uniformity on the range space such that $M$ is uniformly continuous. Conventional definitions of uniformities are not proper to define such a uniformity on the range space.

(9.2) THEOREM. Let $M$ be a mapping from $(S, \mathfrak{U})$ to $R$. Then a unity $\mathfrak{B}=\{\Phi ; \Phi M>\mathfrak{X}\}$ is the strongest unity on $R$ such that $M$ is uniformly continuous.

Proof. Since $\mathfrak{B} M<\mathfrak{U}, M$ is uniformly continuous. Suppose ther is a unity $\mathfrak{C}$ on $R$ such that $M$ is uniformly continuous. Then by the definition of uniform continuity of a mapping $\mathbb{C} M<\mathfrak{A}$, thus every unit of $\mathfrak{C}$ belongs to $\mathfrak{B}$. Therefore $\mathfrak{C} \lessgtr \mathfrak{B}$.

\$ 10. Relation to weak topology. A uniform topology derived from a uniformity $\mathfrak{U}(\mathfrak{U})$ is obviously the same topology derived from con vergence systems defined in $\S 5$. If $\mathfrak{A}$ is a simple unity then it is a weal topology from functions which belong to $\mathfrak{A}$ as units.

(10.1) THEOREM. Let $\mathfrak{I}(\mathfrak{U})$ be a uniform topology derived from a uni formity $\mathfrak{U}(\mathfrak{X})$. Then there exists a unity $\mathfrak{B}$ such that $\mathfrak{A} \sim \mathfrak{B}$ and $\mathfrak{I}(\mathfrak{U})=\mathfrak{I}\left(\mathfrak{B}^{*}\right)$ namely $\mathfrak{I}(\mathfrak{U})$ is a weak topology by functions of $\mathfrak{B}^{*}$.

Proof. For $\Phi \in \mathfrak{A}$ and $a \in S$, define $(f, a)(x)=\sup _{\varphi \in \Phi}|\varphi(x)-\varphi(a)|$. Let $\hat{\Phi}$ be a set of all such functions indexed by $S$. As it has been proved in Lemma (2.1), $\hat{\Phi}$ is equivalent to $\Phi$. Let $\mathfrak{B}$ be a unity of such $\hat{\Phi}$ defined from each $\Phi \in \mathfrak{A}$. Obviously $\mathfrak{U}$ is equivalent to $\mathfrak{B}$. Let $\mathfrak{I}\left(\mathfrak{B}^{*}\right)$ be a weak topology from functions of $\mathfrak{B}^{*}$. Since $y \in U(\Phi, \varepsilon)(x)$ implies $\mid(f, a)(x)-$ $-(f, a)(y) \mid<\varepsilon$ for all $(f, a) \epsilon \hat{\Phi}$, a $\mathfrak{I}\left(\mathfrak{B}^{*}\right)$-open set is $\mathfrak{I}(\mathfrak{H})$-open. Conversely $|(f, x)(x)-(f, x)(y)|<\varepsilon$ implies that

$$
\sup _{p \in \Phi}|p(x)-\phi \cdot(y)|=|(f, x)(x)-(f, x)(y)|<\varepsilon,
$$

thus a $\mathfrak{I}(\mathfrak{U})$ - open set is also $\mathfrak{I}\left(\mathfrak{B}^{*}\right)$-open. Therefore $\mathfrak{I}(\mathfrak{U})=\mathfrak{I}\left(\mathfrak{B}^{*}\right)$.

\section{References}

[1] N. Bourbaki, Éléments de Mathématique II, Livre III, Topologie générate.

[2] E. Hewitt, Rings of real valued continuous functions, II, Trans. Amer. Math. Soc. 64 (1948), pp. 45-99.

[3] J. R. Is bell, Uniform, Spaces, Math. Surveys, 12, 1964.

[4] J. L. Kelley, General topology, Princeton 1955.

[5] S. B. Myers, Functional uniformities, Proc. Amer. Math. Soc. 2 (1951), pp. 153-158.

[6] J. Nagata, On lattice of functions on topological spaces and functions on uniform spaces, Osaka Math. J. 1 (1949), pp. 166-181. 
[7] J. Nagata, A charaeterization of general uniform spaces by a system of uniformly contimuous functions, J. Inst. Polytech. Osaka City Univ., Ser. A, 4 (1953), pp. $43-49$.

[8] H. Nakano, Topology and linear topological spaces, Tolkyo 1951.

[9] - Uniformity and transformation groups, 1968.

[10] J. Tukey, Convergence and uniformity in topology, Princeton 1940.

[11] A. Weil, Sur les espaces à structure uniforme et sur la topologie générale, Actualités Sci. Ind. 551 (1937).

LOYOLA UNIVERSITY

\section{Some Wallman compactifications of locally compact spaces}

by

\author{
H. L. Bentley (Toledo, Ohio)
}

In 1938, Wallman [14] introduced the compactification of a $T_{1}$ space known by his name. In 1964, Frink [4] used a modification of Wallman's procedure to obtain a certain class of compactifications of Tychonof spaces; these compactifications have been called Wallman compactifications. Using the concept of normality of a base $\mathcal{F}$ for the closed sets in a Hausdorff space $X$, Frink constructed a compactification of $X$, denoted by $w(\mathcal{F})$, as the space of all ultrafilters of sets of $\mathcal{F}$. By choosing different bases $\mathcal{F}$ for a non-compact Tychonoff space $X$, different Hausdorff compactifications of $X$ may be obtained in the form of Wallman compactifications $w(\mathcal{F})$. Frink asked whether every Hausdorff compactification can be obtained by this construction. This question remains unanswered; however, many partial results have been obtained: [1], [2] $[3],[5],[6],[7],[9],[10],[11],[12]$, and [13]. Thus, there has been some interest in methods of manufacturing normal bases. This paper is devoted to the study of a general method of constructing such bases in locally compact spaces. Our method is an amplification of the construction of the Alexandroff compactification. As an application of our results, we are able to prove that certain compactifications not previously known to be Wallman are indeed so. For example, we prove that if the remainder in a compactification $Y$ is a certain type of retract of $Y$ then $Y$ is a Wallman compactification.

Throughout this paper, all topological spaces are assumed to be Hausdorff and locally compact; also, we let $X$ denote a fixed locally compact Hausdorff space.

I. The construction of normal bases. Let $\mathcal{F}$ be a family of closed sub sets of the space $X$. $F$ is a ring iff $\mathscr{F}$ is closed under finite unions and intersections. $\mathcal{T}$ is disjunctive iff given any closed set $E$ and any point $x \notin E$, there exists $A \in \mathscr{F}$ such that $x \in A$ and $A \cap E=\emptyset$. $\mathcal{F}$ is normal iff given any two disjoint sets $A_{1}, A_{2} \in \mathcal{F}$ there exist $C_{1}, C_{2} \in \mathcal{F}$ such that $A_{1} \cap C_{1}$ $=\varnothing, A_{2} \cap C_{2}=\varnothing$, and $C_{1} \cup C_{2}=X . \mathscr{F}$ is a normal base iff $\mathscr{F}$ is normal 